\title{
Observations on interphase characterisation in polymer composites by nano-scale indentation using AFM and FEA
}

\author{
T. J. Young ${ }^{1^{\star}}$, L. E. Crocker ${ }^{1}$, W. R. Broughton ${ }^{1}$, S. L. Ogin ${ }^{2}$ and P. A. Smith ${ }^{2}$ \\ ${ }^{1}$ National Physical Laboratory, Hampton Road, Teddington, TW11 0LW, UK \\ ${ }^{2}$ The University of Surrey, Guildford, GU2 7XH, UK. * Tim.young@npl.co.uk
}

\section{SUMMARY}

The possibility of a third phase, often referred to as the interphase, in polymer composites is well known, and there have been many attempts to characterise the mechanical properties of this region at the nanoscale. Despite the increasing resolution of nano-indentation and atomic force microscopy techniques, it is not always possible to identify thin interphases when the dimensions are similar to those of the indenter-surface contact area. The use of indentation for mechanical characterisation continues to be explored for multi-phase systems, and there is a need for a method to evaluate quickly whether a given indentation is influenced by neighbouring material phases. The results presented in this paper demonstrate, both experimentally and through finite element analysis, a method of quickly identifying indentations that are restricted by the reinforcement.

Keywords: A. Polymer-matrix composites (PMCs); B. Interface/interphase; C. Micromechanics; C. Finite element analysis (FEA)

\section{INTRODUCTION}

The existence of an interphase in polymer composites is well known, and there have been many attempts to characterise the mechanical properties of this region at the nanoscale using instrumented nano-indentation testing (IIT) and atomic force microscopy. A limitation of these techniques is that the plastic and/or elastic deformation produced during the indentation may be restricted by the proximity of the reinforcement [1-2]. This leads to transition measurements that relate to the properties of the constituent materials, and obscure the effects due to any interphase which might be attributable to a number of factors, including the presence of a coupling agent. For this reason, the clearest IIT or atomic force microscopy results relating to interphase properties are only for systems with a thick interphase region that is artificially created (with additional coatings on the fibre) [3] or expanded (by water aging) [4].

When the purpose is to characterise the variation in the material properties of an interphase region, rather than the effect of any reinforcement, it is essential that there are methods available to identify when and why interphase measurements are problematic. Current methods tend to rely on calculation of the size of the deformed area [5], comparative measurement of composite systems with and without interphases using a variety of indentation conditions [1] and finite element analysis (FEA) e.g. [6], (although FEA 
approaches do not always model the indenter profile used in the experiments). All of the above methods rely on full characterisation of the indenter geometry and knowledge of the exact location of each indentation with respect to the edge of the reinforcement. Given these issues, it is not surprising that there is some uncertainty regarding the effect of fibre proximity on AFM measurements.

Recent work has shown that it is possible to determine whether indentations are affected by the proximity of the reinforcement [7]. In this work it was suggested that when an indentation takes place across an interface of an inhomogeneous surface, e.g. the glass/resin interface in a glass-fibre polymer composite, there is a lateral force exerted on the atomic force microscope (AFM) tip and an associated torsion of the cantilever. The aim of the current paper is to show that the lateral force, identified by measuring the torsion on the AFM cantilever, may be used to verify, both quickly and unambiguously, whether indentations are representative of the material or restricted by a local reinforcement (i.e. a transition measurement). Results will be presented in the form of experimental and finite element analysis of AFM indentations over the interface of a glass fibre-reinforced phenolic composite in which a lateral force on the AFM tip is identified for all transition measurements.

\section{EXPERIMENTAL METHODS}

\section{Materials}

A phenolic-glass composite consisting of a phenolic resin (Resinox CL1880 mixed with 7 wt\% $\mathrm{H} 1196$ hardener) reinforced with unidirectional E-type glass fibres of $20 \mu \mathrm{m}$ diameter coated with an aminosilane coupling agent was used; the composite was supplied by Dr Alma Hodzic, University of Sheffield, UK. Hodzic et al [4] identified an interphase region of greater than $5 \mu \mathrm{m}$ between the glass fibre and phenolic-resin after water aging for 10 weeks at $23{ }^{\circ} \mathrm{C}$, and the material in the present study was aged in the same way. Hodzic et al [4] found the interphase to have a higher stiffness than the surrounding matrix, which it was argued was a consequence of the coupling agent dispersing into the matrix after water ageing. Recent work [7] has shown that whilst a region of higher stiffness can be measured, its extent is much more limited (approximately $200 \mathrm{~nm}$ ) and the torsion of the indenter tip is dominated by the fibre reinforcement. The surface was prepared for AFM analysis using a modified version of the mechanical polishing method employed by Khanna et al [8] with additional ultrasonic bath treatment.

\section{Instrumentation}

A Park XE-100 (Parks Systems, Suwon, KR) instrument was used for the AFM indentation experiments with a diamond tip of $85 \mathrm{~nm}$ radius, mounted on a $200 \mathrm{~N} \mathrm{~m}^{-1}$ sapphire cantilever, manufactured and calibrated by MicroStar (Huntsville, USA) [12].

During indentation, the torsion of the cantilever was recorded using a LabVIEW (National Instruments, Texas, USA) interface as in [7]. The LabVIEW detection system measured the voltage difference of the photosensitive diode which relates principally to the torsion of the cantilever and lateral force on the indenter tip". 


\section{Experimental Procedure}

The experimental procedure was carried out in the following order. Prior to indentation the AFM tip radius was measured using the SEM and the cantilever spring constant quoted by the supplier was verified. The system was then calibrated by indentation on sapphire, to determine the frame compliance [8], and on reference samples, to determine the effective tip radius [9-10]. The composite samples were indented using an array comprising of four lines of indentations and the elastic modulus was calculated using a Hertzian model [11]. The same reference samples were indented again to check for parameter changes and the tip was examined using the SEM for changes as a result of wear [12].

The AFM experiments produced results for the vertical and torsional forces as a function of indentation depth. The modulus was determined using the analysis outlined below, while the torsional gradient was determined from the linear portion of the torsion-indentation plot.

The reduced elastic modulus was calculated using a Hertzian model (equations 1 and 2 below) with the effective tip radius, $R$, established using the reference samples [9].

$$
\begin{aligned}
& E_{r}=\frac{3 \alpha}{4 \sqrt{R}} \\
& \alpha=\frac{F}{h^{1.5}}
\end{aligned}
$$

In equation 2, $F$ is the force at indentation depth, $h$, and $\alpha$ is a constant.

The reduced elastic modulus, $E_{r}$, is related to the sample elastic modulus, $E_{s}$, by

$$
\frac{1}{E_{r}}=\frac{\left(1-v_{s}^{2}\right)}{E_{s}}+\frac{\left(1-v_{I}^{2}\right)}{E_{I}}
$$

where $E_{l}$ is the indenter elastic modulus, $v_{I}$ is the Poisson's ratio of the indenter and $v_{s}$ is the Poisson's ratio of the sample [9].

\section{Finite Element Analysis}

The overall purpose of the finite element analysis was to simulate the system which had been investigated experimentally for which no interphase region (relating to a coupling agent) had been detected except for a $200 \mathrm{~nm}$ transition region of intermediate stiffness. The FEA analysis modelled contact between the AFM tip and the composite material at various locations to try to ascertain whether the experimentally measured torsion could be produced by indenting in close proximity to the harder reinforcement. The model geometry shown in Figure 1 was created in Abaqus/CAE V6.9-1, the analysis was performed using 
Abaqus/Standard V6.9-1, and the results were generated using Abaqus/CAE. The model was designed as a 3D half hemisphere to reduce the data processing time. The indenter was defined as an analytical rigid surface with an associated reference node. The bulk of the specimen was meshed with 3D continuum elements C3D8R, which are 8 noded linear brick elements with reduced integration and hourglass control. The mesh for the phenolic-glass surface was highly refined in the expected region of contact with the indenter, with the minimum element length being $0.98 \mathrm{~nm}$. The outer layer of elements was meshed using CIN3D8 elements, which are 8 noded linear, one-way infinite elements. One side of the mesh was divided into regions $50 \mathrm{~nm}$ wide so that the material definitions in these regions could be altered easily. This allows the phenolic-glass interface to be 'moved' while keeping indentation occurring in the refined mesh region. Linear elastic material properties were defined for the two materials and yielding and viscoelastic effects were ignored. Contact was modelled between the indenter and the specimen with the rigid indenter defined as the master surface and the deformable mesh as the slave surface. The model was friction-free. Node-to-surface contact was used along with finite-sliding, which allows any arbitrary motion of surfaces. In node-to-surface contact, contact constraint coefficients are generated according to the interpolation functions at the points where the slave node projects onto the master surface. The master surface was smoothed, removing discontinuities which could cause convergence problems. The boundary conditions applied were as follows. The plane of symmetry was constrained using symmetry constraints and the outer nodes of the infinite elements were fully constrained. The reference node of the indenter was constrained so as to allow movement only in the vertical direction. A concentrated load was applied to the reference node. In line with the symmetry condition, only half of the total load was applied. It should also be pointed out that in this FEA analysis, yielding and viscoelastic effects have been ignored.

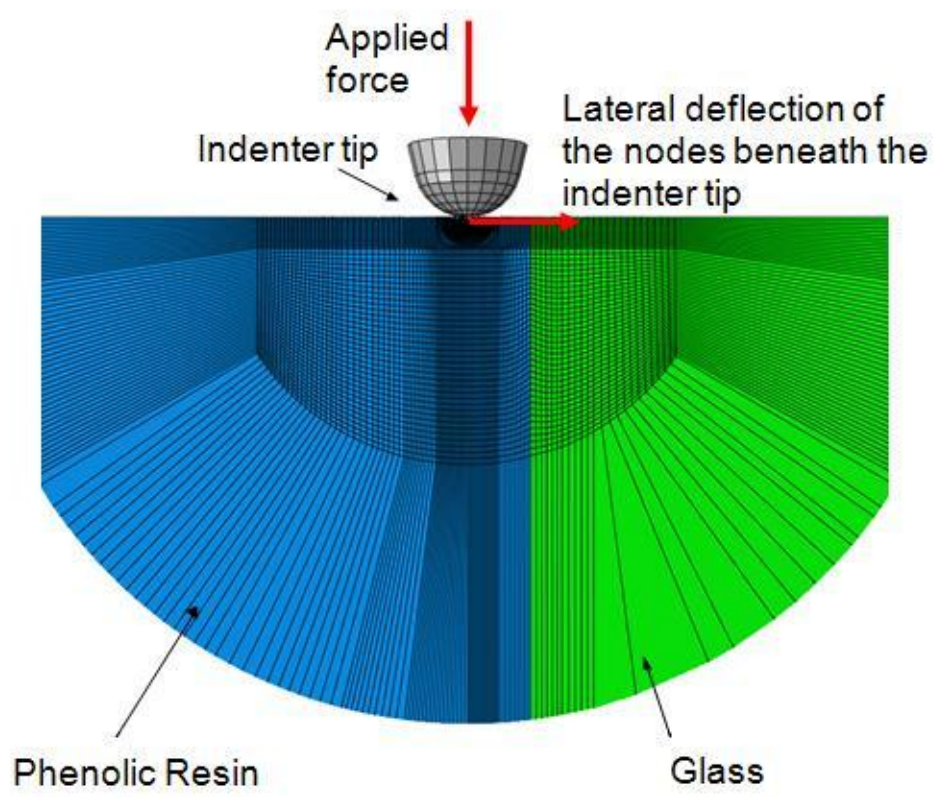

Figure 1: Image of the FEA model used to calculate indentation depth and lateral movement of the tip during simulations of indentation at various distances from the silica reinforcement. 


\section{RESULTS}

Figure 2 shows the force and lateral displacement as a function of indentation depth calculated from the FEA for a range of the indentations. Six indentations were modelled on the phenolic resin at incremental distances from the interface $(25 \mathrm{~nm}$ to $678 \mathrm{~nm})$, one indentation was modelled at the interface $(0 \mathrm{~nm})$ and five indentations were modelled on the silica $(-25 \mathrm{~nm}$ to $-678 \mathrm{~nm})$. From the force-depth curves, it is noticeable that the indentation contact becomes stiffer closer to the interface, suggesting a gradual change in material properties. However, the FEA model is of a perfect interface, with a distinct change in material properties. In addition, for each indentation there is a corresponding lateral displacement of the nodes beneath the indenter tip, and this lateral displacement increases for indentations closer to the interface. This suggests that the reinforcement is restricting the indentation, leading to the apparent increase in stiffness. For the indentation within the phenolic resin at a distance of $25 \mathrm{~nm}$ from the interface there is a change in slope of the lateral displacement/indentation curve at the point where the indenter makes direct contact with the silica reinforcement i.e. at an indentation depth of about $4 \mathrm{~nm}$.
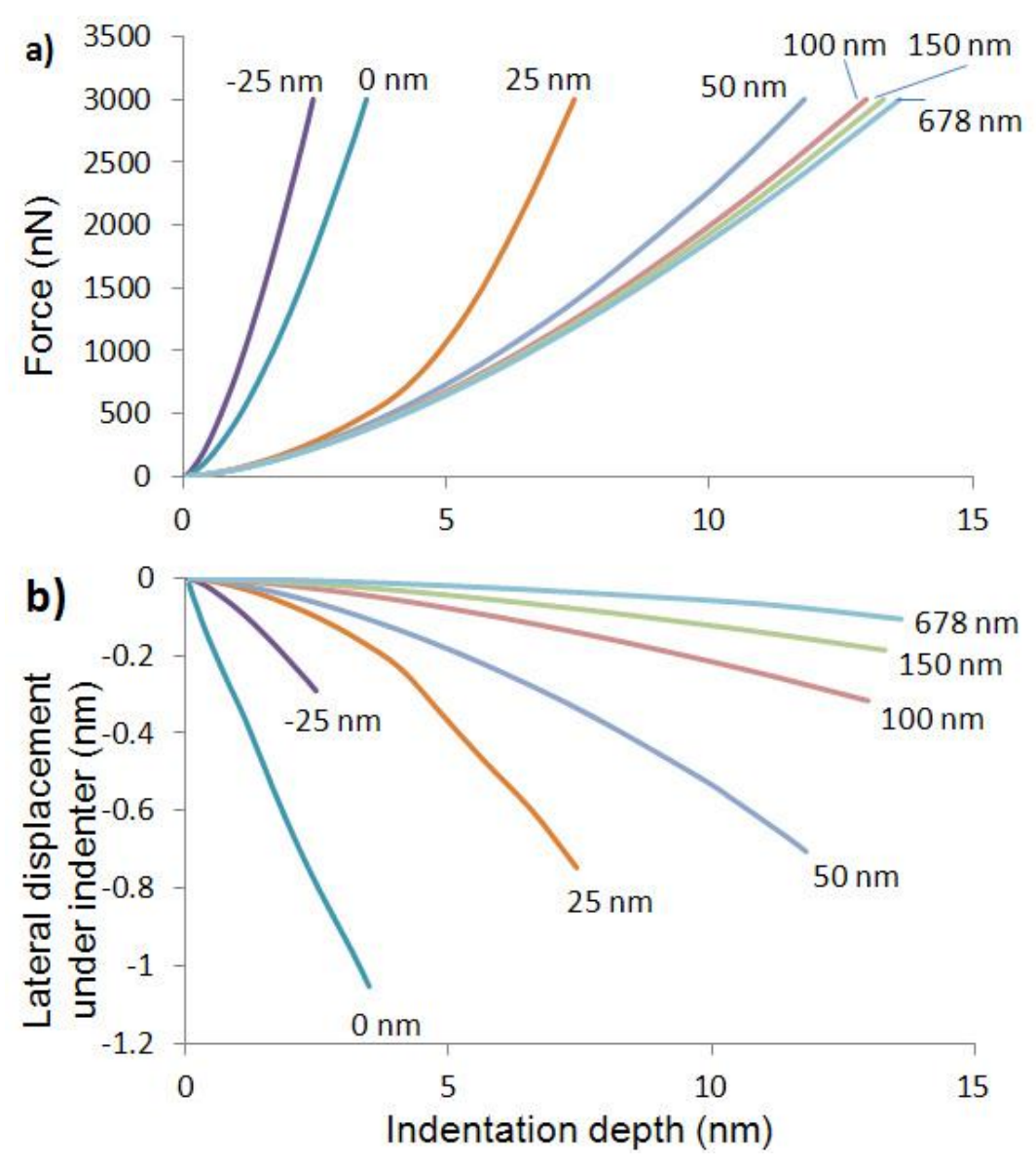

Figure 2: Finite element-based results for a) force as a function of (vertical) indentation depth and b) lateral displacement as a function of (vertical) indentation depth for indents at various distances from the glass fibre surface. 
Table 1 shows the elastic moduli values that were calculated from the FEA model for indentations at various distances from the interface (using equations 1 to 3 ) along with the curve fitting parameters (obtained from the curves in Figure 2). The reduced elastic modulus of the phenolic resin for the indentation modelled (using FEA) at $678 \mathrm{~nm}$ from the interface is then calculated to be $4.5 \mathrm{GPa}$. For an assumed Poisson's ratio of 0.39 (the value used in the FEA model), the elastic modulus of the phenolic resin is calculated to be $3.8 \mathrm{GPa}$, which is the same as the value assumed in the FEA model for the bulk phenolic resin. Based on the results shown in Table 1, it is suggested that interaction starts to become apparent at distances of between 50 and $100 \mathrm{~nm}$ from the interface.

Table 1: Calculated reduced elastic modulus and the associated increase from the bulk sample elastic modulus for the FEA indentations as a function of the proximity to the interface.

\begin{tabular}{|c|c|c|}
\hline $\begin{array}{c}\text { Proximity } \\
\text { to the } \\
\text { interface } \\
\text { (nm) }\end{array}$ & $\begin{array}{c}\text { Curve } \\
\text { fitting } \\
\text { parameter } \\
\boldsymbol{\alpha} \\
\left(F=\alpha h^{1.5}\right)\end{array}$ & $\begin{array}{c}\text { Reduced } \\
\text { elastic } \\
\text { modulus, } \boldsymbol{E}_{\boldsymbol{r}} \\
\text { (GPa) }\end{array}$ \\
\hline-678 & 924.1 & 75.2 \\
\hline-150 & 911.2 & 74.1 \\
\hline-100 & 897.2 & 73.0 \\
\hline-50 & 853.0 & 69.4 \\
\hline-25 & 773.0 & 62.9 \\
\hline 0 & 459.8 & 37.4 \\
\hline 25 & 86.0 & 7.0 \\
\hline 50 & 66.2 & 5.4 \\
\hline 100 & 60.9 & 4.8 \\
\hline 150 & 59.5 & 4.7 \\
\hline 200 & 57.4 & 4.7 \\
\hline 678 & 55.9 & 4.5 \\
\hline
\end{tabular}

Figure $3 a$ shows the reduced elastic moduli values calculated from the FEA results and for four lines of AFM indentations obtained experimentally. Overall, both the FEA and experimental results confirm an increase in the elastic moduli with increasing proximity to the interface, with the modulus increasing from about 4.5 GPa within the phenolic and increasing within a transition region to a value of 65-70 GPa within the glass. The results suggest a transition region of at least $150 \mathrm{~nm}$ where there is a variation of the measured elastic moduli. It is also apparent that the FEA generated value of reduced elastic modulus immediately at the interface is approximately midway (i.e. $\approx 37.4 \mathrm{GPa}$ ) between that of the glass fibrereinforcement (70 GPa) and the phenolic resin $(4.5 \mathrm{GPa})$. This suggests that indentations on the edge of the glass fibre-reinforcement may lead to moduli values that are between that of the fibre and the matrix giving the impression of an interphase. This interpretation is consistent with the suggestions of Gao et al [1] and Munz et al [2] that any transition region within two to three times the indentation diameter is associated with restriction of the 
indentation. The average experimental contact diameters were $87.9 \mathrm{~nm}$ and $31.7 \mathrm{~nm}$ for indentations in the bulk phenolic and glass fibre, which are in good agreement with the FEA modelled indentations of $87.6 \mathrm{~nm}$ and $34.4 \mathrm{~nm}$ for indentations at $678 \mathrm{~nm}$ (phenolic) and $678 \mathrm{~nm}$ (glass).

Figure $3 \mathrm{~b}$ shows the tip torsion gradient measured during the AFM indentations as a function of distance, and the FEA tip deflection gradient as a function of distance calculated from the FEA results in Figure 2. The FEA tip deflection gradients are calculated using a secant value (i.e. the lateral displacement divided by the indentation depth at a peak load of $3 \mu \mathrm{N}$ ). In the FEA model, the indenter is rigid and is constrained laterally which leads to a force on the side of the indenter and corresponding lateral deflection of the nodes beneath the indenter. This is analogous to a deflection of the indenter at the tip apex and given the offset position of the cantilever, an indicator of where there would be torsion of the indenter. Both sets of results indicate a change in the lateral deflection at the indenter tip over the transition region identified in Figure 3a. The FEA results show that the lateral deflections of the indenter found experimentally is not simply a consequence of an interphase but is a consequence of the restriction of the indentation due to the proximity of the reinforcement. Furthermore, the tip deflection gradient can be used to define the position of the indentation in relation to the interface. The FEA results also show that the tip deflection gradient is at a maximum for an indentation at the interface (i.e. $0 \mathrm{~nm}$ ), and near zero for an indentation in the bulk material.

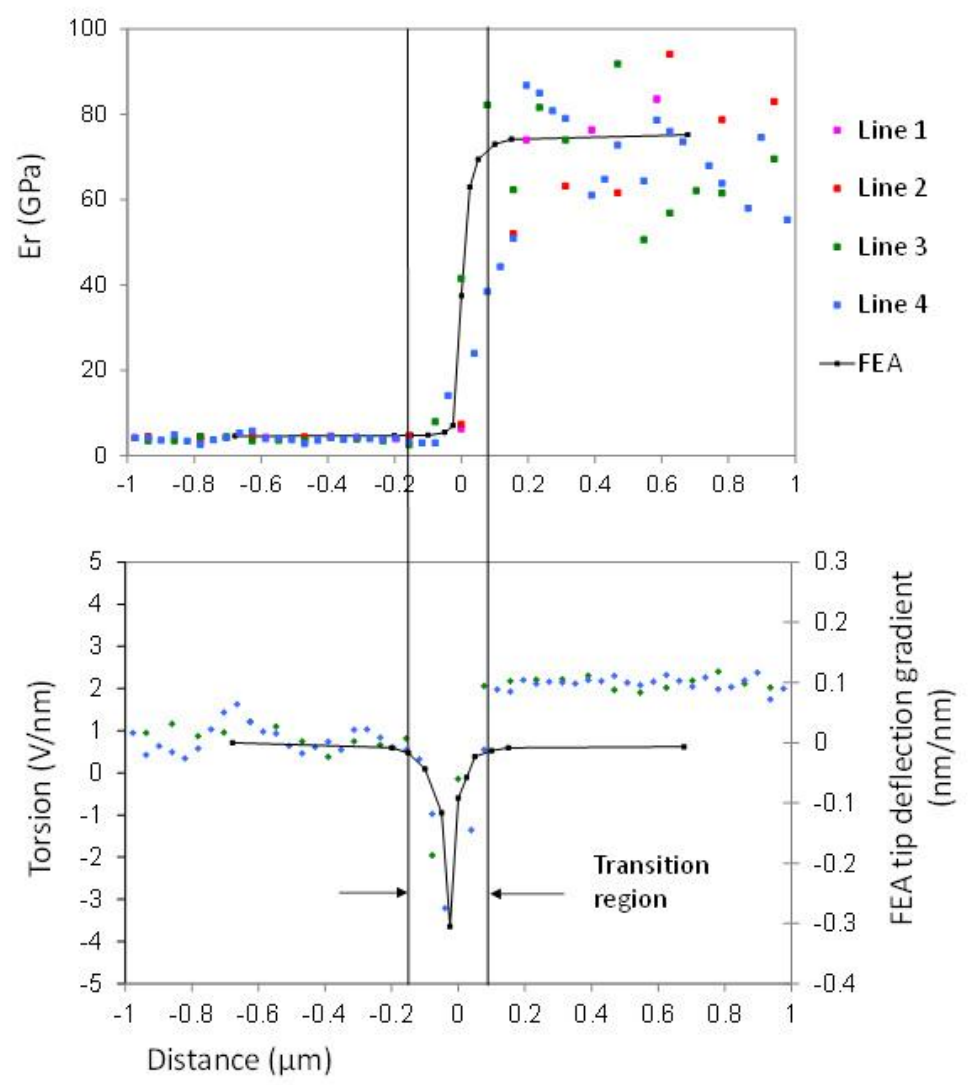

Figure 3: Experimental results obtained using the AFM and output from the FEA model of a) reduced elastic moduli (GPa) and b) the indenter tip lateral deflection gradients. 
The experimental results on these specimens suggest that the interphase, if it exists, is very much smaller than the $5 \mu \mathrm{m}$ measured by Hodzic et al $[4,5]$. which may relate to differences in the water conditioning. The AFM results suggest a transition region of about $150 \mathrm{~nm}$ to $200 \mathrm{~nm}$, which is larger than the transition region indicated by the FEA results (about 100 $\mathrm{nm}$ ), suggesting that the interphase might be about 50 to $100 \mathrm{~nm}$ in extent. However, since there is a significant lateral torsion (or deflection gradient) for each of the indentations within the $200 \mathrm{~nm}$ transition region it is impossible to make a definitive statement about the size of the interphase.

\section{CONCLUDING REMARKS}

The results of the FEA study presented in this paper suggest that a novel method of measuring the torsion on the AFM cantilever (or calculated by FEA), may be used quickly, and unambiguously, to verify whether indentations are representative of the material, or restricted by a local reinforcement. Furthermore, the results suggest that the transition region (even for a perfect interface) may also include indenting into the harder phase which can magnify the apparent size of the interphase.

The results from both AFM indentation and FEA show a correspondence between the torsion and the FEA tip deflection gradient as a function of distance from the interface. The technique of monitoring torsional forces on the AFM tip is therefore proposed as a method of identifying elastic modulus measurement artefacts in the region of a fibre-matrix interface. Furthermore, it is recommended that future studies evaluate the extent of the interphase by subtracting the effect of the transition region (where torsional effects are detected) from the indentation measurements.

\section{ACKNOWLEDGEMENTS}

This work was supported by the National Physical Laboratory as part of a Department for Business, Innovation and Skills (BIS) funded project and an Engineering and Physical Sciences Research Council (EPSRC) funded Engineering Doctorate studentship. The authors would like to thank Dr Miguel Monclus from IMDEA and Dr John Nunn from the National Physical Laboratory for their guidance and support.

\section{REFERENCES}

[1] S. L. Gao and E. Mäder, "Characterisation of interphase nanoscale property variations in glass fibre reinforced polypropylene and epoxy resin composites," Composites Part A: Applied Science and Manufacturing, vol. 33, no. 4, pp. 559-576, 2002.

[2] M. Munz, "Evidence for a three-zone interphase with complex elastic-plastic behaviour: nanoindentation study of an epoxy/thermoplastic composite," Journal of Physics D: Applied Physics, vol. 39, no. 18, pp. 4044-4058, Sep. 2006.

[3] M. R. VanLandingham, R. R. Dagastine, R. F. Eduljee, R. L. McCullough, and J. W. Gillespie, "Characterization of nanoscale property variations in polymer composite systems: 
1. Experimental results," Composites Part A: Applied Science and Manufacturing, vol. 30, no. 1, pp. 75-83, 1999.

[4] A. Hodzic, J. K. Kim, and Z. H. Stachurski, "Nano-indentation and nano-scratch of polymer/glass interfaces. II: model of interphases in water aged composite materials," Polymer, vol. 42, no. 13, pp. 5701-5710, 2001.

[5] A. Hodzic, Z. H. Stachurski, and J. K. Kim, "Nano-indentation of polymer-glass interfaces Part I. Experimental and mechanical analysis," Polymer, vol. 41, no. 18, pp. 6895-6905, 2000.

[6] S. H. Lee, S. Wang, G. M. Pharr, and H. Xu, "Evaluation of interphase properties in a cellulose fiber-reinforced polypropylene composite by nanoindentation and finite element analysis," Composites Part A: Applied Science and Manufacturing, vol. 38, no. 6, pp. 15171524, 2007.

[7] T. J. Young, M. A. Monclus, Broughton, W. R., Ogin, S. L., and Smith, P.A., "Observations on interphase characterisation in polymer composites by nano-scale indentation and the use of AFM cantilever torsion to identify measurement artefacts," Plastics, Rubber and Composites. Available online 2012, Retrieved from http://www.ingentaconnect.com/content/maney/prc/pre-prints.

[8] S. Khanna, R. Winter, P. Ranganathan, S. Yedla, M. Kalukanimuttam, and K. Paruchuri, "Sample preparation techniques for nano-mechanical characterization of glass fiber reinforced polyester matrix composites," Composites Part A: Applied Science and Manufacturing, vol. 34, no. 1, pp. 53-65, Jan. 2003.

[9] M. Salerno and I. Bykov, "TUTORIAL: Mapping Adhesion Forces and Calculating Elasticity in Contact-Mode AFM," Microscopy and Analysis SPM supplement, John Wiley and Sons, Itd, 2006.

[10] C. A. Clifford and M. P. Seah, "Quantification issues in the identification of nanoscale regions of homopolymers using modulus measurement via AFM nanoindentation," Applied Surface Science, vol. 252, no. 5, pp. 1915-1933, 2005.

[11] M. A. Monclus, T. J. Young, and D. Di Maio, "AFM indentation method used for elastic modulus characterization of interfaces and thin layers," Journal of Materials Science, vol. 45, no. 12, pp. 3190-3197, Mar. 2010.

[12] B. Mesa and S. Magonov, "Novel diamond/sapphire probes for scanning probe microscopy applications," Journal of Physics: Conference Series, vol. 61, pp. 770-774, 2007.

[13] D. C. Lin, E. K. Dimitriadis, and F. Horkay, "Robust Strategies for Automated AFM Force Curve Analysis-I. Non-adhesive Indentation of Soft, Inhomogeneous Materials," Journal of Biomechanical Engineering, vol. 129, no. 3, p. 430, 2007.

[14] Applied scanning probe methods VII: Biomimetics and industrial applications. Berlin: Springer, 2007. 